\title{
Intensionality in mathematics: problems and prospects
}

\section{Introduction to the special issue}

\author{
Marianna Antonutti Marfori ${ }^{1}$. Paula Quinon ${ }^{2}$
}

Published online: 8 March 2021

(c) The Author(s) 2021

This volume aims to explore philosophical issues related to intensionality in mathematics, and critically examine how concerns about intensionality have driven much recent work in both philosophy and mathematics, particularly in formal philosophy and mathematical logic.

Intensional issues, in mathematics and elsewhere, are often most easily characterised in contrast to extensional ones. Consider the case of algorithms: two algorithms may have quite different descriptions, and indeed quite different characteristics - one may run in linear time, while the other has merely polynomial running time - and yet compute the same outputs. According to a purely extensional standard they would thus be considered identical, since they compute the same function, but intensionally they are distinct. Set theory, on the other hand, is often considered a paradigmatic case of an extensional discipline: the fact that two sets have the same members is sufficient for them to be identical, regardless of how those sets are described, and indeed this principle is codified in axiomatic set theories such as ZFC by the axiom of extensionality.

These examples indicate how intensional approaches to mathematics can usefully be characterised in terms of their treatment of the notion of identity. If the identity of two items (terms, sentences, functions, proofs, etc.) can be established by examining the objects that fall under their extensions, then the approach in question is an extensional one. On the other hand, if establishing identity requires considering additional aspects that are connected to the way in which such items are defined or presented, then the approach is intensional. (Consider for example the question of whether two algorithms which compute the same function can be considered to be the same algorithm) In other words, we can say (in a somewhat circular manner) that if an approach does not distinguish between two different presentations of the same object or concept,

$凶 \quad$ Marianna Antonutti Marfori

Marianna.AntonuttiMarfori@1rz.uni-muenchen.de

1 Munich Center for Mathematical Philosophy, Ludwig-Maximilians-Universität München, Munich, Germany

2 Warsaw University of Technology, Faculty of Administration and Social Sciences, International Center for Formal Ontology, Warsaw, Poland 
then it is extensional. If instead it does not treat as equivalent an object or concept when it is presented by different descriptions, then the approach is intensional. (This characterisation is circular because it presupposes that we already know when two differently-presented objects or concepts are the same object or concept, which is sometimes not a trivial issue in mathematics.) Intensionality in mathematics is thus traditionally understood as concerning the way in which mathematical objects are presented to us, and how the way in which we represent those objects affects what we can know about them. It is thus apparent how intensionality pervades our language and our reasoning in all mathematical domains, and consequently how difficult it is to encompass the wide range of ways in which it can manifest itself into a single definition.

In the context of mathematical enquiry, adding intensional dimensions to formal or mathematical theories often involves crossing sub-disciplinary borders. For example, if we are working within an extensional approach to functions defined as sets of ordered pairs of inputs and outputs, we may want to add some finer grained information on them by using tools from computability theory or computer science that allow us to look at the way in which the functions are defined or presented, e.g. by characterising the algorithms in terms of the complexity of their definitions. Sometimes, we may also want to bring extra-theoretical resources to bear on intensional issues: for example, when we single out the intended model for a given theory, we may want to take into account how faithful the formalised model is to our informal conception of the semantic domain in question.

Because there is no single overarching "question of intensionality" about a formal system, mathematical model, or mathematical theory, the intensional questions that arise are often addressed in either a piecemeal way, or not at all. It is therefore important to reflect on intensionality in mathematics in a cross-disciplinary way. By addressing questions about intensionality explicitly and comparing solutions across disciplinary boundaries, much progress can be made in understanding intensional issues in mathematics. In turn, such an analysis can provide important insights into mathematics, mathematical reasoning, and mathematical practice.

Explicit philosophical reflection on intensionality in the recent debate (say, the last half century) made notable appearances in logical works from the ' 60 s (see especially early works by Kreisel and Feferman) and featured prominently in the '80s (see especially Feferman's paper and Shapiro's collected volume, both entitled "Intensionality in Mathematics" and dating to 1985). In the last three decades, however, systematic reflection on intensional issues has not occupied a significant role in the philosophy of mathematics and logic, even though at the same time intensional concerns can be seen as driving research in many areas of mathematics and logic. To give but a few examples, consider the incredible development in fields such as intensional logics and semantics, and computability and complexity theory, over the last half century.

This volume was conceived with the goal of drawing more attention to the importance that intensional issues play in contemporary research in mathematics and logic, and promote explicit discussion of intensionality, with the belief that this would lay the grounds for achieving progress in identifying common methodological aspects that underpin research in many diverse areas that are driven by intensional concerns. The papers collected in this volume were conceived with the aim of highlighting specific 
intensional concerns that arise in their respective domains of research, and the interdisciplinary components that are often required to address them. Due to their sheer variety, this volume can only do justice to a few of the concerns that fall under the broad umbrella of intensionality in mathematics, with the hope of initiating a larger discussion about these issues and developing some promising new lines of inquiry into their solution.

The idea for this volume came after a conference entitled "Intensionality in Mathematics" organised by the guest editors at the University of Lund on 11-12 May 2013, and generously funded by the Erik Och Gurli Hultengrens Fond för Filosofi. The current volume collects the papers of some of the invited speakers at the conference, and some that were written especially for this project.

The volume opens with a paper by Barbara Sarnecka, who examines the formation of the concept of natural number in young children. Starting from the research framework developed by Carey in The Origin of Concepts (2009), she presents a model of the different stages of number acquisition, based on experimental results. In this model, the core cognitive resources (humans can assess or subitize quantities of up to five elements without counting), and the culturally acquired knowledge of the counting list ("one", "two", "three" etc.) and counting routine (reciting the number list and pointing to objects, one at a time) enable children to grasp the cardinal meaning of numbers and form a placeholder structure. So even if children have an innate ability to represent small sets exactly and large numbers approximately, they gain the ability to represent large, exact cardinal values only when they learn a counting list representation of the natural numbers.

Jan Heylen's paper contributes to the debate about the canonicity of numerical expressions, such as Hindu-Arabic numerals, or arithmetical expressions like "three times four", and how such expressions relate to the possibility of knowledge about numbers. Canonicity is what accounts for the intensionally privileged status of the Peano numerals as natural number notations: in more formal terms, this can be stated as the fact that Peano numerals are eligible for universal generalisation and existential quantification in doxastic contexts, while, many argue, other number names are not. According to Heylen, the canonicity of Peano numerals is explained by results in firstorder epistemic Peano Arithmetic with the addition of a knowledge operator and the full induction scheme in its standard interpretation, where the induction scheme (including occurrences of the knowledge operator) figures as the explanandum. It follows from this that what qualifies as canonical depends on the form of the induction scheme that is adopted. Furthermore, Heylen argues, since Peano numerals are buck stoppers in Kripke's sense that no further calculation or proof is needed to determine their referent, all arithmetical terms that can be reduced to numerals are eligible for possible de re knowledge.

Paula Quinon discusses the merits of the methodology of Carnapian explication for studying intensional differences between formalisations of mathematical concepts by focusing on the case of Church's analysis of the notion of computation in contrast to Turing's. While some have argued that Turing's model provides the correct conceptual analysis of the pre-systematic concept of effective calculability, and others that Church's analysis is the correct one, both sides agree that there can only be one correct conceptual analysis of effective calculability. In contrast, Quinon argues that 
both Church's and Turing's analyses can be considered as successful Carnapian explications of the pre-systematic concept of effective calculability. In doing so, she shows how to overcome an objection of Carnap's to the use of semiformal, semi-interpreted axiomatic systems (such as the axioms for general recursive functions) as explications of pre-systematic mathematical concepts.

Dustin Tucker addresses a family of intensional paradoxes involving propositions and propositional attitudes first studied by Arthur Prior. Such paradoxes involve propositions that themselves embed propositional attitudes: for example, the proposition that everything you hope is false. Tucker formulates a new paradox in this family, the hopefear paradox, and argues that it and similar intensional paradoxes are not susceptible to many of the standard solutions proposed for sentential paradoxes like the Liar, including truth-value gap, contextualist, situation-theoretic, revision-theoretic, ramified, and (possibly) dialetheist approaches. This is because these approaches avoid paradox by denying us the very conceptual resources that we make use of in theorising about them. Tucker goes on to evaluate four approaches that he considers more promising, including classical and non-classical indeterminacy accounts, restricting our propositional attitudes, and the use of free logic.

Gil Sagi provides a new account of logical terms, as terms whose extensions are determined purely by their form. By developing insights from Barcan Marcus's distinction of levels of meaning according to their degree of intensionality, Sagi proposes distinguishing a new level of meaning which is to be situated below that of extensions, and which concerns only structure, i.e. a more coarse-grained, less intensional level of meaning that is appropriate for logic and that is labelled form. The notion of form is then characterised semantically: since forms are invariant under permutations, they are logical notions in the Tarskian sense. According to this account, then, logicality can be shown to be a lower-level analogue of rigidity, and purely logical languages can be characterised as sub-extensional in the sense of being only concerned with form.

Sara Negri examines Stone representation theorems, which represent algebraic structures in terms of set-theoretic structures (often with a topology). In the metatheory of philosophical logic, one of the chief uses of Stone-type representations is proving the faithfulness of translations between logics, for example in the proof of the faithfulness of the translation of intuitionistic logic into the modal logic S4. The algebraic part of this proof consists in the representation of Heyting algebras as the open sets of topological Boolean algebras; the use of these representations makes the proof indirect and non-constructive. While establishing correspondences between systems with respect to derivability, such semantic methods do not show us how to translate a proof from one system to the other. Since, however, logical embeddings can be obtained as translations of derivations in a direct way that does not employ the non-constructive principles typical of Stone-type representation theorems, Negri argues that the use of proof-theoretic methods allows us to recover the intensional, pointfree part of representation results that is not available in traditional point-set topologies.

Luca Tranchini proposes an inferentialist account of meaning based on a new approach to proof-theoretic harmony, the idea that what can be inferred from a logically complex sentence by means of the elimination rules for its main connective should match what needs to be established to infer the sentence in question by using the introduction rules for its main connective. After distinguishing intensional and 
extensional approaches to harmony, Tranchini proposes a new account of harmony based on reductions and expansions by developing a pattern for expansions for arbitrary connectives. The account is then shown to qualify as intensional, in contrast to most alternative accounts, which are based on pairing inversion principles with the inter-derivability of rules, and which Tranchini shows do not qualify as intensional accounts.

Sofie Angere's paper considers the notion of identity in Univalent Foundations, a promising field that has recently been expanding very fast, but is nonetheless still not well understood and not easily accessible to non-specialists. Angere studies the interplay between the intensional aspects of Univalent Foundations deriving from the use of Martin-Löf type theory, which forms the logical basis of Univalent Foundations, and extensional aspects of other logical systems such as modal logic and category theory. This is done by analysing syntactic and semantic aspects of the notions of identity available in Univalent Foundations, in order to clarify the notions of intensionality and extensionality that are relevant in this context. For example, in the case of semantics, a detailed analysis is carried out of Homotopy Type Theory (HoTT), which provides a topological semantics for the type theory used in Univalent Foundations, in contrast to the model-theoretic semantics used for set theory.

Last but not least, we would like to thank the people and institutions without whose work and support this project would not have been possible. Our thanks go to Gila Sher, who spoke at the Lund conference and was then one of the editors in chief of this Journal, for suggesting that we submit a proposal for a special issue of Synthese, and to Otávio Bueno, our corresponding editor in chief, for providing insightful advice and excellent guidance for this project at all and only the times that it was needed. We would like to extend our deep thanks to the authors, who contributed outstanding papers to this volume, and to the referees, who worked alongside the authors and the guest editors to ensure the quality of the volume. Finally, Marianna Antonutti Marfori would like to acknowledge generous support from the German Research Foundation (DFG), Project Number 390218268 (Gefördert durch die Deutsche Forschungsgemeinschaft (DFG)_Projektnummer 390218268), and Paula Quinon would like to thank generous funding from the Seventh Framework Programme of the European Community under the Marie Skłodowska-Curie Grant Agreement No. 301470.

Funding Open Access funding enabled and organized by Projekt DEAL.

Open Access This article is licensed under a Creative Commons Attribution 4.0 International License, which permits use, sharing, adaptation, distribution and reproduction in any medium or format, as long as you give appropriate credit to the original author(s) and the source, provide a link to the Creative Commons licence, and indicate if changes were made. The images or other third party material in this article are included in the article's Creative Commons licence, unless indicated otherwise in a credit line to the material. If material is not included in the article's Creative Commons licence and your intended use is not permitted by statutory regulation or exceeds the permitted use, you will need to obtain permission directly from the copyright holder. To view a copy of this licence, visit http://creativecommons.org/licenses/by/4.0/.

Publisher's Note Springer Nature remains neutral with regard to jurisdictional claims in published maps and institutional affiliations. 